\title{
Modelo de negocios para comercialización de películas y recubrimientos comestibles en Bucaramanga
}

Business model for marketing films and coatings groceries in Bucaramanga 


\title{
Modelo de negocios para comercialización de películas y recubrimientos comestibles en Bucaramanga ${ }^{1}$ Business model for marketing films and coatings groceries in Bucaramanga
}

\author{
Tilcia Yaneth Ardila Pinto², Helga Constanza Uribe Quintero ${ }^{3}$ \\ 2,3Universidad Autónoma de Bucaramanga, Colombia
}

Artículo recibido en enero de 2017; artículo aceptado en marzo de 2017

Citación del artículo: Ardila, Y. \& Uribe, H. (2017). Modelo de negocios para comercialización de películas y

recubrimientos comestibles en Bucaramanga. I+D Revista de Investigaciones, 10(2), julio - diciembre de 2017 , pp. 14 - 24

\begin{abstract}
Resumen
El uso de películas y recubrimientos comestibles busca proteger los alimentos de contaminantes externos y de su proceso natural de descomposición, a fin de extender su vida útil en las estanterías. Esta práctica ha crecido en países como China y España a partir de procesos de investigación, desarrollo e innovación en la generación de biopolímeros naturales, utilizando materias primas de procedencia vegetal o animal (Romero \& Estrada, 2011). En Colombia se han realizado estudios en el ámbito universitario, entre los que se destacan algunos estudios técnicos para la creación de nuevos tipos de películas y recubrimientos. La propuesta de modelo de negocios advierte que en Bucaramanga los empresarios del sector de alimentos requieren comprender esta tecnología antes de incorporarla y desarrollar proyectos que les permitan ser más competitivos en mercados internacionales. El enfoque de esta investigación es exploratorio y la muestra ha sido seleccionada por conveniencia; el trabajo de recolección de información está soportado con entrevistas aplicadas en profundidad a expertos de empresas del sector de alimentos de la región. El análisis de los resultados muestra situaciones en común de la industria agropecuaria que hacen inviable el modelo de negocio para esta ciudad debido al bajo interés (del sector y de la industria) en la inversión para proyectos de investigación y desarrollo.
\end{abstract}

Palabras clave: comercialización, películas, recubrimientos, comestibles, modelo de negocios.

\footnotetext{
Abstract

Films and edible coatings are practical to protect food from external contaminants and their natural process of decomposition looking to extend their shelf life. This practice has been growing in countries like China and Spain from processes of research, development and innovation in the generation of natural biopolymers, using raw vegetable or animal origin materials. In Colombia there have been studies in universities, highlighting technical studies to

1. Artículo investigativo con enfoque cualitativo, resultado de un proyecto de investigación para definir un modelo de comercialización, en la Maestría de Administración de Empresas (culminado) desarrollado en el Grupo de Investigación de Mercados y Negocios Internacionales - Grimeni de la UNAB y financiado por la Universidad Autónoma de Bucaramanga, (Colombia). Dirección: Avenida 42 No. 48 - 11, PBX: 6436111. Fecha de inicio: noviembre de 2015, fecha de terminación: mayo de 2017.

2. Ingeniero de Mercados, Universidad Autónoma de Bucaramanga - UNAB. MBA Administración de Empresas, con profundización en Marketing, Universidad Autónoma de Bucaramanga. Grupo: Grimeni. Universidad Autónoma de Bucaramanga, (Colombia). Dirección: Avenida 42 No. 48 - 11 , PBX: 6436111. Orcid: 0000-0001-9109-4062. Correo institucional: tardila@unab.edu.co.

3. Ingeniero de Mercados, Universidad Cooperativa de Colombia - UCC. MBA Administración de Empresas, con profundización en Marketing, Universidad Autónoma de Bucaramanga - UNAB. Universidad Autónoma de Bucaramanga (Colombia). Dirección: Avenida 42 No. 48 - 11, PBX: 6436111. Orcid: 0000-0003-3040-9205. Correo institucional: huribe@unab.edu.co.
} 
create new types of films and coatings. The proposed business model, warns that in Bucaramanga entrepreneurs in the food sector, need to understand this technology, sensitizing them to develop projects, enabling them to be more competitive in international markets. The focus of this research is exploratory and the sample selected for convenience; supported depth interviews applied with experts from companies in the food sector. The interview is structured with open questions. In the analysis of the arguments of the respondents, situations common agricultural industry are identified, throwing as a result the non-viability of the business model for this city, for its low interest in investment for research and development projects.

Keywords: marketing, films, coatings, comestible, business model.

Según el Centro Tecnológico de Soluciones para tu Innovación [Ainia] (2013), en la actualidad los consumidores están valorando alimentos variados, sanos, con buen sabor, de calidad superior y listos para consumir, que les generen mayor bienestar, tanto en cuanto a su salud como en la practicidad a la hora de preparar la comida. Para dar respuesta a esta necesidad se han venido estudiando varias tecnologías, entre las que se encuentran las películas de recubrimiento comestible, que ayudan a preservar la vida útil de los alimentos sin que se pierdan sus propiedades nutricionales y sus características sensoriales. De acuerdo al Plan de Desarrollo de Santander (2016), en donde se advierte que se trabajará para que Santander logre posicionarse como la tercera economía más fuerte del país para el año 2030, buscando el fortalecimiento de la competitividad de la región (Gobernación de Santander, 2016). El propósito de este proyecto es participar del crecimiento regional a partir del desarrollo de tecnologías de empacado basado en películas y recubrimientos de tipo comestible, inicialmente en Bucaramanga y su Área Metropolitana, como una alternativa para la conservación de alimentos y siendo responsables con el medio ambiente.

La ingeniera Ximena Carrión Granda desarrolló las películas y recubrimientos comestibles a partir del quitosano y la proteína del suero de la leche; utilizando, además aceites esenciales de tomillo y romero, y extracto de salvia, ajo y romero, que al contacto con los mariscos y pescados ayuda a extender su vida útil y permite reducir los microorganismos deteriorantes. Este desarrollo fue probado en catorce tipos de bacterias que afectan directamente a este tipo de proteínas, obteniendo los mejores resultados en atún fresco, merluza y langostinos (Carrión, 2016a).

En la Universidad de Navarra se han desarrollado otros estudios documentados en tesis de estudios de posgrado, como las tesis "Desarrollo, caracterización y aplicaciones alimentarias de películas comestibles basadas en la zeina de maíz" de Mendoza García (2009), y "Películas biodegradables activas a base de quitosano: desarrollo y caracterización de sus propiedades tecnológicas y su aplicación en la protección de semillas de alcachofa" (Ziani, 2008).
De acuerdo con Embuscado y Kerry (2009), en los últimos años ha venido creciendo el mercado de las películas y recubrimientos comestibles, aunque se debe tener en cuenta que este tipo de productos han sido estudiados desde siglos atrás como alternativas de protección y durabilidad de los alimentos a partir de componentes de origen vegetal o animal. Esta, por supuesto, es apenas una solución entre las que se han venido dando en la industria de los alimentos.

Frente a este panorama, se pretende identificar el mercado potencial para este tipo de tecnología, advirtiendo que la implementación de dicha tecnología obedece a desarrollos de la organización Proinec, empresa española dedicada a la investigación, desarrollo e innovación (I+D+i) de materiales basados en biopolímeros de tipo natural, extraídos de diferentes productos alimenticios o naturales, que permiten proteger los alimentos de contaminantes externos y de su mismo proceso natural de descomposición y así extender su vida útil en las estanterías (Bellón, 2010).

En la actualidad, en Bucaramanga la conservación de los alimentos utiliza materiales y tecnologías tradicionales que afectan el medio ambiente y no permiten la conservación adecuada de los productos, en este sentido las películas y recubrimientos comestibles se presentan como la oportunidad de comercializar una tecnología más avanzada. De acuerdo con lo anterior, y teniendo en cuenta el enfoque agroindustrial de la región, la investigación busca responder a la pregunta: ¿Cuál debería ser el modelo de negocios adecuado para la comercialización de proyectos de investigación o de películas y recubrimientos comestibles, en el sector agroindustrial de Bucaramanga?

En este sentido, el proyecto propone establecer si los empresarios del sector agroindustrial de Bucaramanga están dispuestos a adquirir las películas y recubrimientos ya fabricados que son amigables con el medio ambiente, o si alguna empresa de dicho sector está dispuesta a desarrollar la tecnología de películas y recubrimientos adaptados especialmente a sus negocios. 


\section{Método}

\section{Tipo de estudio}

El enfoque de esta investigación es de tipo exploratorio; el estudio se realizó en Bucaramanga y su Área Metropolitana con el fin de conocer el uso de películas y recubrimientos comestibles, el conocimiento que los empresarios vinculados a la actividad de producción de alimento tienen sobre la tecnología en estudio y su aplicabilidad en el sector agroindustrial de la región.

\section{Enfoque cualitativo}

Al considerarse la utilización de entrevistas a profundidad como herramienta de recolección de información, se advierte que la investigación tiene una orientación cualitativa, ello debido a que se busca a partir de la aplicación del instrumento el entendimiento que empresarios vinculados a la producción de alimentos que se encuentran en la región tienen sobre la tecnología de películas de recubrimientos comestibles, como una opción para mejorar la conservación de los alimentos dispuestos para la venta al público. Además comprender la percepción sobre generación de valor a partir de la incorporación de procesos de I+D+i que son pertinentes y/o necesarios en su entorno empresarial, así como la disposición en participar y acceder a recursos de gobierno que son destinados para este fin.

\section{Participantes}

La muestra se seleccionó a través de muestreo no probabilístico a juicio, considerando dos empresas del sector avícola de acuerdo a su relevancia en el sector económico de la región y el país, adicionalmente, se consultó con una empresa dedicada a la fabricación y comercialización de dulces tradicionales dentro y fuera del país. Es importante resaltar que los empresarios entrevistados son expertos en métodos de conservación de cada uno de estos sectores, según Vanguardia Liberal, 2016.

\section{Materiales e instrumentos}

El instrumento utilizado para la recolección de la información está conformado por entrevistas en profundidad aplicadas a expertos vinculados a empresas del sector de alimentos en Bucaramanga y su área metropolitana. Las entrevistas están conformadas por preguntas abiertas que abordaron temas de conocimiento, intencionalidad, disposición y requerimientos frente a la utilización de películas y recubrimientos comestibles para la conservación de sus productos.

\section{Entrevista}

Los expertos fueron entrevistados personalmente. En la Tabla 1 se definen las variables consideradas en el proceso de la entrevista.

Tabla 1

Variables del estudio

\begin{tabular}{ll}
\hline Necesidad & Descripción \\
\hline Innovación & $\begin{array}{l}\text { Posibilidad de nuevos } \\
\text { desarrollos en las compañías }\end{array}$ \\
Métodos de empacado & $\begin{array}{l}\text { Procesos de empacado } \\
\text { utilizados por las compañías }\end{array}$ \\
Métodos de conservación & $\begin{array}{l}\text { Procesos de conservación de los } \\
\text { productos }\end{array}$ \\
Inversión en I\&D+i & $\begin{array}{l}\text { Presupuesto económico } \\
\text { asignado para I\&D+i }\end{array}$ \\
Producción & $\begin{array}{l}\text { Capacidad de producción en } \\
\text { toneladas por día }\end{array}$ \\
Conocimiento & $\begin{array}{l}\text { Nivel de actualización en nuevas } \\
\text { tecnologías }\end{array}$ \\
Personal & $\begin{array}{l}\text { Personal capacitado para la } \\
\text { I\&D+i }\end{array}$ \\
\hline
\end{tabular}

Fuente: Autores

\section{Procedimiento}

Una vez realizadas las entrevistas, se resalta que la industria en Bucaramanga y su área metropolitana no invierte en desarrollo o innovaciones en empaques investigativos que generen inversiones adicionales a lo netamente importante para ellos, que es la innovación y desarrollo de sus productos terminados. Además, resaltan que son los proveedores de empaques quienes realizan los procesos de $I \& D+i$ en sus productos con el fin de llegar al empresario con nuevas alternativas que cumplan con los requerimientos de protección, contención y promoción de los alimentos en los que se utilizan. Aunque advierten que en ciertas circunstancias son ellos los que determinan las características del tipo de empaque que requieren, pero no hacen procesos de investigación conjunta para obtener un nuevo tipo de empaque.

Con la experiencia en el mercado, las industrias entrevistadas han controlado sus procesos evitando 
problemas en sus productos, y en este momento no es de su interés extender la vida útil de sus productos ya que como lo están haciendo mantienen la frescura, la calidad y se cumple con sus expectativas comerciales y lo que esperan los consumidores.

Las industrias cumplen con la ley colombiana con respecto a la conservación del medio ambiente, y a los procesos industriales de los productos que ofrecen al mercado.

En los sectores entrevistados es importante el manejo de los costos en su producción, para lograr utilidades positivas al finalizar su ejercicio, igual que para todas las industrias, es decir, que controlan costos, además de sus gastos. Importante mencionar que el consumidor no valora las inversiones en empaques o medios de conservación del producto, para ellos está inherente la calidad con la marca.

\section{Modelo de negocio}

Con base en la información recopilada se planteó un modelo de negocio donde se presenta, además de su estructura básica, el entorno necesario para que el modelo tenga éxito en la generación de servicios asociados a proyectos de investigación o comercialización de películas y recubrimientos comestibles, de acuerdo con los requerimientos identificados en las empresas de la industria de alimentos en Bucaramanga y su área metropolitana.

\section{Plan de negocio}

Según Publishing (2007), un plan de negocio se define como las acciones futuras que la empresa debe ejecutar, utilizando los recursos con los que cuenta, para lograr determinados objetivosymetas. Una de sus características es que debe ser plasmado en un documento físico, que será el soporte para establecer el plan de comunicación interna y externa. Otras características de la definición y generación de un modelo de negocio son el sentido lógico, el enfoque progresivo, la mirada realista, la coherencia de su estructura y accionar, y su orientación a la acción.

Según Borello (2000), el plan de negocios se denomina también plan económico y financiero, ya que se describe como un instrumento para un proceso sistemático y eficaz. La profundidad del plan de negocio, depende del tamaño y estructura de la empresa, así como la estructuración del plan depende de la experiencia gerencial o técnica de los directivos que conforman dicha empresa. El plan de negocio incluye un análisis del mercado, del sector y de la competencia y requiere de una actualización constante. Por su parte, Stutely (2000) define el plan de negocios como un plan en el que se plasma la metodología que permitirá llevar a cabo una actividad en cierto periodo de tiempo.

Según Borello (2000) el plan de negocio es útil ya sea para determinar la factibilidad de inversión, la definición de la actividad empresarial o en la gestión de la actividad empresarial, y es importante des de el inicio de la empresa hasta la búsqueda de estrategias para su crecimiento y consolidación.

La metodología para generación de modelos de negocios más usada en la actualidad es la desarrollada por Osterwalder y Pigneur (2011), conocida como metodología Canvas, la cual será la base para estructurar el modelo propuesto para la prestación de servicios de I+D+i, y para la comercialización de películas y recubrimiento comestibles en Bucaramanga y su área metropolitana.

En la Tabla 2 se detalla la metodología a seguir para elaborar la propuesta del modelo de negocios según Alexander Osterwalder, y en la Tabla 2 se presenta el lienzo diligenciado como propuesta para este modelo de negocio.

\section{Oportunidad de negocio}

El foco de oportunidad parte de las exigencias de mercados desarrollados en segmentos de preservación de alimentos, en donde es viable la creación de un modelo de negocio dedicado a comercializar proyectos de investigación para el sector alimenticio en Bucaramanga y su área metropolitana a partir del desarrollo de películas y recubrimientos comestibles; una nueva empresa en Bucaramanga, dedicada al desarrollo de películas y recubrimientos comestibles, sería un proveedor empresarial asociado a la generación de impactos positivos con el medio ambiente mediante la utilización de materiales amigables en procesos de conservación de alimentos, buscando extender la vida útil de los alimentos y mejorando su aspecto físico específicamente en productos de exportación, estando a la vanguardia de tecnologías en los temas expuestos con anterioridad.

\section{Mercado objetivo}

Considerando que la utilización de películas y recubrimiento comestibles puede acondicionarse a la conservación de alimentos frescos y procesados, se identifica que el mercado objetivo se concentra 
Tabla 2

Propuesta de modelo de negocios para la comercialización de películas y recubrimientos comestibles en empresas de Bucaramanga

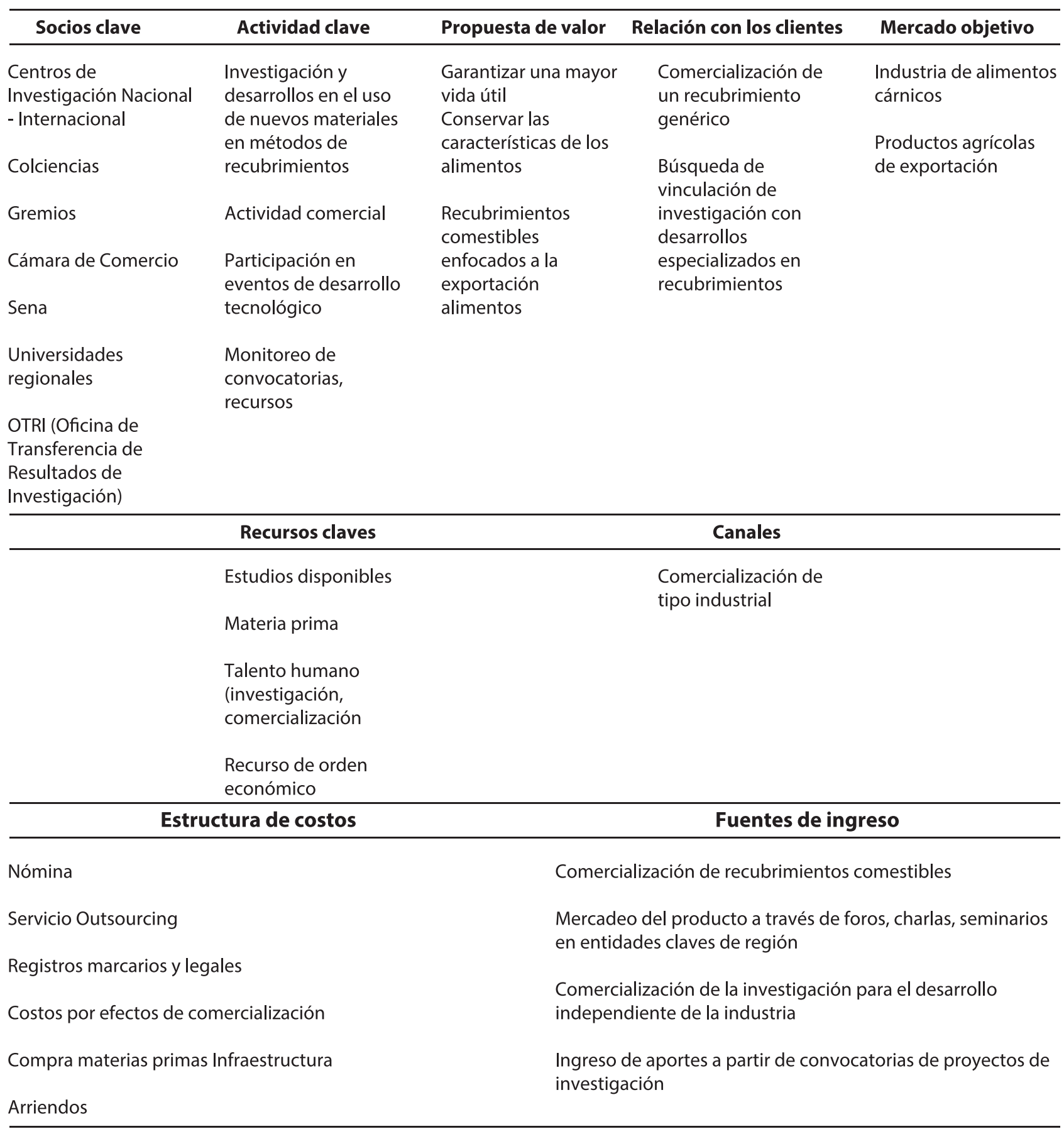

\section{Fuente: Autores}


en empresas que se preocupan por procesos de conservación y empacado amigables y efectivos, y que le brinden un mayor tiempo de vida a los productos en el mercado. Adicionalmente, y teniendo en cuenta que es una tecnología novedosa, y que diversos alimentos requieren de barreras más resistentes en sus procesos de conservación, debido a sus características, algunas empresas proponen al público objetivo productos con generación de valor a partir del desarrollo asociado a conservación de alimentos.

Los resultados del estudio realizado dejan ver la negación de las empresas de la región en acceder a proyectos de investigación con base en recursos del gobierno, tipo convocatorias realizadas por Colciencias o Innpulsa para obtener recursos para la investigación y desarrollo de nuevos productos; debido a que consideran que son más los costos que los beneficios económicos y tecnológicos que se puedan obtener, por lo cual el proceso de I\&D+i es un componente dentro del modelo de negocio que no deberá estar limitado a la disposición de las organizaciones o entidades gubernamentales, sino que por el contrario deberá ser un factor proactivo que conduzca a generar valor en productos terminados y listos para comercializarse de acuerdo a los requerimientos de los productos de la industria de alimentos. Siendo así, el modelo de negocios sugiere entender las particularidades de cada alimento, iniciar los procesos de investigación para desarrollar la película de recubrimiento más adecuada para el alimento y posteriormente, iniciar los procesos de comercialización en empresas que producen y comercializan dichos alimentos y que utilizan materiales de conservación tradicionales o diferentes al desarrollo propuesto por el nuevo modelo de negocios.

\section{Canales de comercialización}

La estrategia de comercialización se basa en el hecho de que el target es altamente especializado, lo que conlleva que se realicen contactos directos a partir de encuentros programados con las empresas y con los diversos públicos que hacen parte de ellas, considerando que cada contacto es crítico para los procesos de comercialización. En este contexto existe la probabilidad de que una organización requiera dos o tres presentaciones previas antes de poder iniciar de manera formal el proceso de comercialización del desarrollo de la película o recubrimiento, siendo considerados como relevantes quienes toman la decisión y quienes advierten las bondades del desarrollo de películas de recubrimientos comestibles frente a los requerimientos del producto (alimento) que produce la empresa. En ese orden, los expertos técnicos, los encargados de procesos de conservación, empaque o envasado, y el gerente de la empresa serán quienes decidan -en las presentaciones formales del modelo de negocios- si se ejecuta o no.

En otra instancia y si los empresarios deciden no desarrollar esta tecnología para sus productos, la estrategia para comercializar películas y recubrimientos comestibles será la importación de las películas para venderlas de forma genérica para la industria del sector de alimentos, a través de un modelo de distribución directa.

\section{Relaciones con los clientes}

La relación con los clientes debe ser directa y personalizada, ello debido a que, si bien el proceso de conservación de alimentos es similar entre sí, las características y requerimientos de cada organización son potencialmente diferentes, convirtiéndose en el factor de éxito poder desarrollar y comercializar películas de recubrimiento ajustadas a las particularidades de cada producto del portafolio de las organizaciones vinculadas a la industria agropecuaria de Bucaramanga y su área metropolitana. De igual forma, los procesos encaminados a generar proyectos de $1 \& D+i$ estarán sujetos a especificaciones técnicas a partir de nuevos requerimientos empresariales, posteriores a tener una relación definida basada en la confianza entre el proveedor (nuevo modelo de negocios) y la empresa de producción de alimentos para el desarrollo y la aplicabilidad de las películas y recubrimientos comestibles. Aunque la relación inicial se concentra en ámbitos transaccionales, la intención conduce a que el proceso permanezca en el tiempo y derive en proyectos conjuntos de nuevos desarrollos tecnológicos con beneficios mutuos. Para la materialización de los proyectos de I\&D+i es importante trabajar de la mano con centros de investigación que tengan la capacidad de soportar estudios detallados dirigidos a obtener nuevos desarrollos asociados a la conservación de alimentos, de acuerdo con las necesidades de la industria y la aplicabilidad de las películas y recubrimientos en sus productos.

Es evidente también, considerando los resultados obtenidos en la investigación realizada, que el proceso de comunicación enfocado en concientizar a las empresas de la industria de alimentos para aprovechar los recursos gubernamentales que apoyan los proyectos de I\&D+i y así obtener beneficios tributarios, puede ser una fuente interesante de recursos que servirían para aumentar la competitividad de cada empresa, teniendo ventajas competitivas en el mercado y generando nuevas soluciones a los públicos que atiende. 


\section{Estructura de ingresos}

La generación de recursos económicos asociados a ingresos se ve representada en:

- La comercialización de películas y recubrimientos comestibles ya desarrollados y de acuerdo a requerimientos de ciertos productos de la industria de alimentos.

- La gestión de proyectos conjuntos con empresas del sector alimentos, buscando la generación de clúster que permitan realizar I\&D+i relacionados con conservación de alimentos a partir de la utilización de películas de recubrimiento comestibles, repercutiría en una mayor disposición de los empresarios para desarrollar está tecnología de una manera más económica y motivante para la aplicación en sus productos terminados. Este tipo de proyectos generalmente reciben aportes de entidades del Estado como Colciencias.

\section{Actividades clave}

Entre las actividades claves para la gestión de la empresa se sugieren temas relacionados con comercialización, operación en investigación y gestión en el relacionamiento con centros de investigación que puedan soportar nuevos desarrollos tecnológicos, resaltando actividades como:

En I\&D+i:

- Monitoreo de fuentes de recursos públicas y privadas asociadas a la generación de proyectos de investigación relacionados con formas de conservación de alimentos.

- Alianzas con centros de investigación regionales que apoyen los procesos de desarrollo de películas de recubrimiento comestibles.

- Investigación asociada a validar nuevos desarrollos divulgados en productos o procesos de conservación de alimentos.

En promoción del nuevo modelo de negocios:

- Participación en eventos de desarrollo tecnológico asociados a la industria de alimentos.

- Relaciones públicas para tener presencia en actividades asociadas a nuevas tecnologías y tendencias en la industria de alimentos.
En comercialización de películas de recubrimiento comestibles:

- Identificación de empresas prospecto y concreción de reuniones de presentación de la oferta del nuevo modelo de negocios.

- Identificación de requerimientos técnicos asociados a conservación de alimentos.

- Identificación de requerimientos para la entrada a nuevos mercados de productos alimenticios producidos en Bucaramanga y su área metropolitana.

\section{Recursos clave}

Siendo un nuevo modelo de negocios que parte de procesos de $1 \& D+i$ en la conservación de alimentos, se identifican recursos claves asociados a la gestión del conocimiento tecnológico desde el ámbito de la investigación. En este sentido, que el modelo de negocios pueda tener desarrollos tecnológicos que se vean respaldados por algún tipo de protección legal, se convierte en un recurso clave que soporta tanto el reconocimiento como las actividades que pueda llegar a desarrollar el modelo. Junto con la tecnología desarrollada como parte de los recursos claves, el talento humano vinculado con la organización (técnico y comercial) es vital en el buen ejercicio del modelo.

\section{Red de aliados estratégicos}

Para que el modelo de negocios pueda llegar a ejecutarse se requiere de la conformación de una red de aliados que conduzca a un crecimiento rápido, un posicionamiento paulatino y una adecuada gestión en generación de negocios, lo cual se logra considerando los aportes que instituciones aliadas puedan brindar al modelo. Por lo anterior, la presencia de centros de investigación nacional e internacional enfocados en el desarrollo de producto y procesos de conservación de alimentos como las películas de recubrimiento comestibles son indispensables. En el contexto regional, la presencia de centros de investigación vinculados a instituciones universitarias comola Universidad Industrial deSantander -UIS, y la Universidad Autónoma de Bucaramanga -UNAB, posibilita tener aliados estratégicos interesantes en la validación de requerimientos para proyectos de investigación, validación de requerimientos técnicos para conservación de alimentos y desarrollo de nuevas tecnologías.

Desde el ámbito internacional, la presencia de Proinec como un desarrollador de productos asociados a recubrimientos para conservación de alimentos con 
experiencia en Europa, permite tener un aval que se manifiesta en la posibilidad de comercializar sus productos en la región y facilitar la concreción de negocios en el entorno regional.

La cercanía con ciertas asociaciones o gremios puede facilitar los procesos de investigación colaborativa y de comercialización, siendo los más relevantes la Sociedad de Agricultores de Santander, la Federación Nacional de Avicultores de Colombia (Fenavi) y la Federación Colombiana de Ganaderos (Fedegan).

\section{Estructura de costos}

La estructura de costos de este nuevo modelo de negocios puede definirse inicialmente en aspectos propios de su operación investigativa y productiva, su gestión comercial y de mercadeo, así como su relación con diferentes organizaciones que conduzcan a la generación de recursos.

- Nómina

- Servicio de externalización

- Registros marcarios y legales

- Costos por efectos de comercialización

- Compra de materias primas

- Arriendos

\section{Resultados}

Con base en el estudio realizado, se encontró que las industrias del sector de alimentos en Bucaramanga y su área metropolitana cuentan con personal calificado en los procesos de desarrollo, investigación e innovación y se dedican a estudiar constantemente las tendencias del mercado para lograr ofrecer, a través de su tecnología, productos de alta calidad, y que los consumidores demandan y valoran. Adicionalmente, y basados en la experiencia con entidades dedicadas a realizar aportes económicos, las industrias que invierten prefieren utilizar recursos propios en sus proyectos de innovación y así evitar la tramitología requerida para obtener los recursos de entidades que convocan a participar en proyectos subsidiados por el Estado. Las grandes industrias de Santander que realizan un aporte considerable al PIB tanto de la región como del país, cuentan con economías integradas verticalmente, que les permiten ser más productivas y rentables en sus negocios.

Los proveedores tradicionales de materiales como las láminas o películas utilizadas por la industria alimenticia son los que invierten en investigación, desarrollo e innovación en empaques que genere inversiones, la industria de alimentos, se dedica a lo netamente importante para ellos, que es la innovación y desarrollo de sus productos terminados.

Con la experiencia en el mercado, las industrias entrevistadas han controlado con sus procesos los problemas de calidad y conservación de sus productos, y no es de su interés extender la vida útil de los mismos ya que como lo están haciendo mantienen la frescura, la calidad y se cumple con sus expectativas comerciales y lo que esperan los consumidores. Además, las industrias cumplen con la ley colombiana con respecto a la conservación del medio ambiente y a los procesos industriales de los productos que ofrecen al mercado.

En las empresas estudiadas, igual que para todas las industrias, es fundamental controlar los costos relacionados con la producción de sus marcas; en este proyecto es importante mencionar que las empresas consideran que el consumidor no valora las inversiones en empaques o medios de conservación del producto, pues para ellos la calidad es inherente a la marca, por tanto lo que se generaría para las industrias de alimentos sería un mayor costo en su producción, que no se recuperaría en la venta.

Las industrias cuentan con la experiencia en el desarrollo de nuevos productos, investigación e innovaciones con expertos externos, que los han apoyado en nuevos proyectos, por lo cual argumentan que no están interesadas en invertir en proyectos investigativos para la aplicación de nuevas tecnologías, ya sea directamente o a través de terceros, como entidades gubernamentales, así ofrezcan beneficios tributarios, por cuanto tendrían, de todos modos, que invertir en ellos para llevarlos a la realidad, y no cuentan con los recursos necesarios.

\section{Discusión}

Los resultados del estudio realizado indican que las empresas de la región no están interesadas en acceder, a partir de proyectos de investigación, a recursos del Gobierno, debido a que consideran que los costos superan los beneficios económicos y tecnológicos que se puedan obtener; por esta razón el proceso de I\&D+i es un componente dentro del modelo de negocio que no deberá estar limitado a la disposición de las organizaciones, sino que, por el contrario, deberá ser un factor proactivo que conduzca a generar valor en productos terminados y listos para comercializarse de acuerdo a los requerimientos de los productos de la industria de alimentos. Siendo así, el modelo de negocios sugiere que primero deberá entender las particularidades de cada alimento, iniciar los procesos de investigación para desarrollar la película de recubrimiento más 
adecuada para el alimento y posteriormente, iniciar los procesos de promoción y comercialización en empresas que producen y comercializan dichos alimentos y que utilizan materiales de conservación tradicionales o diferentes a la nueva tecnología basada en películas y recubrimientos comestibles, propuesto por el nuevo modelo de negocios.

\section{Conclusiones}

La investigación permitió determinar que el tamaño de mercado para películas y recubrimientos comestibles en Bucaramanga es amplio, teniendo en cuenta la variedad y número de empresas dedicadas a la producción de alimentos en la región; por ello, se considera que se tendría opción para aplicar esta nueva tecnología, además que la economía bumanguesa es la cuarta del país, y cuenta con grandes industrias en el sector de alimentos que aportan al PIB nacional.

Con esta investigación se conocieron los diferentes métodos empleados para la conservación de alimentos por la industria alimentaria de Bucaramanga, y las necesidades de inversión de estas empresas en proyectos de innovación y desarrollo; a partir de ello se concluyó que, en la actualidad, el sector de alimentos de Bucaramanga y su área metropolitana no hace uso de las películas y recubrimientos comestibles de última tecnología en sus procesos de conservación.

Por otra parte, se concluye que la industria representativa del sector primario de la región no involucra en sus procesos de inversión en innovación a los "ángeles inversores" con los que cuenta el país, ni se interesa en los beneficios tributarios que estos le ofrecen.

Se identifica, con este proyecto, que la ciudad de Bucaramanga y en general la región no cuenta con muchos centros de investigación que sean del interés de los empresarios para realizar estudios y desarrollos de investigación que puedan cubrir la demanda de su sector empresarial.

Adicionalmente, se concluye que los sectores alimenticios de la región ignoran este tipo de tecnología de conservación, y que les parecería interesante conocerla más a fondo y poder aplicarla sin tener que hacer mayores inversiones.

Asimismo, se concluye que las empresas de la industria de alimentos de Bucaramanga y su área metropolitana se encuentran a la vanguardia en métodos de conservación, y que cubren sus necesidades de conservación de sus alimentos sin requerir, por ahora, el desarrollo de una nueva infraestructura para la aplicación de nuevas tecnologías en conservación de alimentos, basadas en innovaciones y desarrollos propios. Se advierte, no obstante, que la industria está dispuesta a aplicar nuevas tecnologías ya desarrolladas.

Con base en el contacto realizado con el pionero de esta tecnología en España, se pudo determinar que por ahora no es de su interés establecer relaciones comerciales a través de canales de distribución directa en Colombia, bajo ninguna modalidad.

\section{Recomendaciones}

Se sugiere que alguna institución educativa, como la Universidad Autónoma de Bucaramanga, asuma un rol de liderazgo para generar una alianza estratégica entre los centros de investigación, ya existentes, y la industria, buscando motivar y apoyar nuevos desarrollos de innovación basados en investigación, y generando mayor confianza en la integración academia e industria.

Sensibilizar a la academia y a la industria acerca de la importancia de trabajar conjuntamente para lograr el desarrollo de la región, algo que traerá beneficios competitivos y de crecimiento y permitirá cumplir uno de los objetivos planteados por el gobierno local, como es hacer de Santander la tercera economía del país.

Motivar a los centros de investigación para hacer alianzas estratégicas con pioneros o desarrolladores de esta tecnología en otros países o universidades.

\section{Agradecimientos}

Agradecemos a la Universidad Autónoma de Bucaramanga, y en especial al doctor Edgar Mauricio Mendoza García, pionero de este tipo de tecnología en su doctorado en la Universidad de Navarra, en España, por sus valiosos aportes y el conocimiento transmitido para el desarrollo de este proyecto. De igual forma, agradecemos a nuestro director de proyecto, $\mathrm{Mg}$. Adolfo Segura Moya, del Programa de Ingeniería de Mercados, por su orientación, conocimiento, asesoría, tiempo y dedicación desde el inicio y hasta el final de este proyecto.

\section{Referencias}

Ainia. (2013). ¿Recubrimientos comestibles en alimentos? ¿para que? Recuperado de http://www.ainia.es/noticias/ alimentacion-saludable/recubrimientos-comestibles-enalimentos-para-que/

Alcaldía de Bucaramanga. (2014). Planeación de ordenamiento territorial de Bucaramanga. Bucaramanga. 
Bellón, M. (2010). Proinec investigará recubrimientos comestibles para la industria alimentaria. Alimarket.

Borello, A. (2000). El plan de negocios: de herramienta de evaluación de una inversión a elaboración de un plan estratégico y operativo. McGraw-Hill Interamericana.

Cámara de Comercio de Bucaramanga. (2014a). Producto Interno Bruto Santander. Bucaramanga.

Cámara de Comercio de Bucaramanga. (2014b). Compite360.

Carrión Granda, X. (11 de abril de 2016a). Recubrimientos comestibles desarrollados en a UPNA permiten extender la vida útil de pescados y mariscos. Universidad Pública de Navarra. Recuperado de http://www.unavarra.es/ actualidad/noticias?contentld $=220172$

Durango, M., Soares, N. (2013). Avances en alimentos y sus tendencias. 9(135).

Embuscado, E. \& Kerry, H. (2009). Edible Films and Coatings for Food Applications. New York: Springer Editor.

Fedegán. (2015). Página del Ganadero Fondo Nacional del Ganado FNG. Obtenido de http://static.fedegan.org.co/ notas/PG_13032016.pdf

Gobernación de Santander. (2016). Plan Desarrollo Santander. Recuperado de http://www.santanderinnova.org.co/ media/3dd2981d782b90f18ce03bc9822c45ed.pdf

Mendoza, E. (2009). Desarrollo, caracterización y aplicaciones alimentarias de películas comestibles basadas en zeína de maíz. Universidad Pública de Navarra.

Osterwalder, A. \& Pigneur, Y. (2011). Generación de modelos de negocio. Barcelona: Grupo Planeta.

Publishing, M. (2007). El plan de negocios. Ediciones Días de Santos.

Romero, L. \& Estrada, J. (2011). Elaboración de una película comestible a base de colágeno incorporado con nisina como agente antimicrobiano para reducir la pérdida de humedad y oxidación de las grasas en filetes de carne de cerdo en refrigeración. Cartagena, Colombia.

Stutely, R. (2000). Plan de negocios: la estrategia inteligente. México: Prentice Hall.

Vanguardia Liberal. (2016). Las 500 Empresas Generadoras de Desarrollo en Santander. (G. S.A.S., Ed.) Recuperado de http://www.vanguardia.com/empresasgeneradoras/

Ziani, K. (2008). Películas biodegradables activas a base de quitosano: desarrollo y caracterización de sus propiedades tecnológicas y su aplicación en la protección de semillas de alcachofa. Universidad Pública de Navarra. 